University of Wollongong

Research Online

Faculty of Social Sciences - Papers (Archive) Faculty of Arts, Social Sciences \& Humanities

2016

Length of stay as a predictor of reliable change in psychological recovery and well being following residential substance abuse treatment

Brie Turner

University of Wollongong, blt75@uowmail.edu.au

Frank P. Deane

University of Wollongong, fdeane@uow.edu.au

Follow this and additional works at: https://ro.uow.edu.au/sspapers

Part of the Education Commons, and the Social and Behavioral Sciences Commons

Research Online is the open access institutional repository for the University of Wollongong. For further information contact the UOW Library: research-pubs@uow.edu.au 


\title{
Length of stay as a predictor of reliable change in psychological recovery and well being following residential substance abuse treatment
}

\author{
Abstract \\ Purpose Longer length of stay (LOS) in residential drug and alcohol treatment has been associated with \\ more favourable outcomes, but the optimal duration has yet to be determined for reliable change indices. \\ Optimal durations are likely a function of participant and problem characteristics. The current study aims \\ to determine whether LOS in a residential therapeutic drug and alcohol treatment community \\ independently predicts reliable change across a range of psychological recovery and well being \\ measures.. Design/methodology/approach Three hundred and eighty clients from Australian Salvation \\ Army residential drug and alcohol treatment facilities were assessed at intake and three months post- \\ discharge using the Addiction Severity Index 5th ed., the Depression, Anxiety and Stress Scale, the \\ Recovery Assessment Scale, the Mental Health Continuum- Short Form and the Life Engagement Test. \\ Findings The findings confirm LOS as an independent predictor of reliable change on measures of well \\ being and client perceived assessment of recovery. The mean LOS that differentiated reliable change \\ from no improvement was 37.37 days. Originality/value The finding of LOS as a predictor of reliable \\ change and the identification of an estimated time requirement may be useful for residential drug \\ treatment providers in modifying treatment durations.
}

\section{Keywords}

treatment, change, abuse, reliable, predictor, stay, length, substance, residential, following, being, well, recovery, psychological

\section{Disciplines}

Education | Social and Behavioral Sciences

\section{Publication Details}

Turner, B. \& Deane, F. P. (2016). Length of stay as a predictor of reliable change in psychological recovery and well being following residential substance abuse treatment. Therapeutic Communities: the international journal for therapeutic communities, 37 (3). 


\begin{abstract}
Objective: Longer length of stay (LOS) in residential drug and alcohol treatment has been associated with more favourable outcomes, but the optimal duration has yet to be determined for reliable change indices. The current study aims to determine whether LOS in residential drug and alcohol treatment independently predicts reliable change across a range of psychological recovery and well-being measures.
\end{abstract}

Method: Three hundred and eighty clients from Australian Salvation Army residential drug and alcohol treatment facilities were assessed at intake and three months post-discharge using the Addiction Severity Index, the Depression, Anxiety and Stress Scale, the Recovery Assessment Scale, the Mental Health Continuum- Short Form and the Life Engagement Test. Results: The findings confirm LOS as an independent predictor of reliable change on measures of well-being and client perceived assessment of recovery. The mean LOS that differentiated reliable change from no improvement was 37.37 days.

Conclusions: The finding of LOS as a predictor of reliable change and the identification of an estimated time requirement may be useful for residential drug treatment providers in modifying treatment durations.

Key Words: Residential treatment, length of stay, drug and alcohol, reliable change 
Length of stay as a predictor of reliable change in psychological recovery and well-being following residential substance abuse treatment.

Length of stay and outcome in residential substance abuse treatment

Residential programs have an important role in the treatment of substance misuse problems globally (Teeson, Mills, Ross, Darke, Williamson \& Havard, 2007; Gossop, 1995). It has been repeatedly argued that the effectiveness of residential drug and alcohol treatment is closely associated with length of stay (LOS) in the program (Gossop et al., 1999; Simpson et al., 1999). Strong positive relationships between LOS and outcomes in residential drug and alcohol treatment have been consistently reported (Teeson, et al., 2007; Greenfield et al., 2004). More often than not, "the best results are seen among those who spent long periods in a single enrolment” (Darke et al., 2012, p. 65).

It has been further proposed that clients must stay in treatment for a minimum of three months to gain significant improvements (Simpson et al., 1999; Ernst \& Young, 1996). This recommendation has led to the three month follow-up period becoming something of a standard (Gossop et al., 1999; Condelli \& Hubbard, 1994), yet, the empirical basis for this is limited. The retention thresholds appear to have been chosen based on the approximation of such periods to average treatment durations in a particular modality, or the schedule of data collection, rather than through examination of alternative intervals (Zhang et al., 2002; Simpson, Joe \& Brown, 1997). Furthermore, there is evidence to suggest improvements are observed in those who leave before this timeframe (Gossop et al., 1999).

Unsurprisingly, LOS is a major determinant of cost of treatment in residential settings (Greenfield et al., 2004). Due to increasing concerns regarding the containment of treatment cost, residential programs that require long tenure are facing mounting pressures to demonstrate that the added cost of longer treatment translates to added benefits in relation to improved client outcomes. As a result, additional studies using continuous measures of LOS 
are necessary to further examine the optimal treatment duration required to produce positive outcomes.

Predictors of Client Outcome

Although the average treatment durations may vary, most studies indicate that clients who remain in treatment for longer periods show more favourable post-treatment outcomes in relation to substance use, employment and criminality than their short stay counterparts (Teeson et al., 2015; Zhang et al., 2002; Gossop et al., 1999; Condelli and Hubbard, 1997). Studies using multivariate analyses have confirmed the predictive utility of LOS in determining treatment outcomes whilst controlling for other potential predictive factors (Zhang et al., 2002; Gossop et al., 1999; Condelli and Hubbard, 1997).

Clients who identify as alcohol users demonstrate better substance use outcomes when compared to those using cocaine or multiple drugs (Hambley \&_Arbour, 2010; Miller et al., 1990). Examination of variables associated with retention in residential treatment has confirmed the predictive utility of primary substance of abuse, with those reporting alcohol as their primary drug having a greater likelihood of remaining in treatment beyond three-months when compared to those with opiates or "other" drugs as their substance of chief concern (Deane, Wootton, Hsu \& Kelly, 2012). Similarly, those presenting with cocaine use have been found to have shorter stays when compared to an alcohol control group (Choi, Adams, Morse \& MacMaster, 2015; Simpson et al., 1997).Types of substances used appear related to length of stay and dropout in a range of alcohol and other drug treatment services and suggest the need to consider them as a potential factor for understanding differences in length of stay and outcome.

The severity of client's drug use patterns has been reliably associated with poorer retention in treatment and subsequently more rapid relapse to substance use following treatment (Simpson et al., 1999; Ryan, Plant \& O’Malley, 1995). Those exhibiting greater 
problem severity at treatment intake generally demonstrate poorer outcomes post-discharge and overtime (Mulder et al., 2009; Simpson et al., 1999).

Finally, gender and age differences have also be related to differential treatment outcomes with most studies indicating women tending to have more positive outcomes than men (Green et al., 2004; Weisner et al., 2003) and older clients (55+) also having more favourable outcomes with the latter being associated with greater LOS (Satre et al., 2004; Mertens and Weisner, 2000).

Most of the substance abuse treatment literature uses abstinence as the primary outcome of treatment and there is no paucity of research to confirm the positive effects of LOS on this outcome (e.g., Teeson et al., 2015; Zhang et al., 2002; Gossop, et al., 1999; Simpson et al., 1999; Condelli \& Hubbard, 1997;). The lack of psychological outcome measures across studies is surprising due to the relatively high prevalence of mental health issues observed in those who abuse substances (Lai, Cleary, Sitharthan \& Hunt, 2015). Furthermore, the Substance Abuse and Mental Health Services Administration includes aspects of psychological and social well-being in their definition of recovery from substance use disorders (SUD) (del Vecchio, 2012). Despite the fact that many drug treatment providers now offer integrated mental health treatment services, the impact of these programs on mental health has been somewhat neglected.

\section{Reliable change}

Statistical significance and effect size statistics have often been used to describe outcome (Teeson et al., 2015; Condelli \& Hubbard, 1997). Yet, these methods are limited in the extent to which they consider the reliability of the instruments of interest (Eisen et al., 2007). The Reliable Change Index (RCI) was developed as an extension of statistical significance testing to provide a measure of statistical significance which takes into account the scale reliability (Christensen and Mendoza, 1986). Thus, change observed using the RCI 
indicates a shift significant enough in magnitude that it is unlikely to be due to measurement error (Eisen et al., 2007).

Much of the available research regarding the RCI focuses primarily on examination of treatment outcome in non-substance using populations. (Gonda et al., 2012; Newnham et al., 2007) and there is a need to utilise these methods in alcohol and other drug treatment contexts.

Aims

The current study aims to identify whether LOS in residential drug and alcohol treatment predicts reliable change on a series of psychological recovery measures above and beyond other participant factors (e.g. age, gender, primary substance of abuse and problem severity). Whilst much research has examined LOS and its relationship to treatment outcome, contention exists regarding the treatment duration required to produce positive outcomes. Given this, the second aim of this study is to explore an estimate of the treatment 'dose' necessary to make reliable change.

\section{Method}

\section{Setting and program description}

The Salvation Army operates eight residential drug and alcohol treatment facilities across three states and territories along the east coast of Australia. The Recovery Service Centres provide up to 10 months of residential treatment in the form of a modified therapeutic community for individuals with a SUD. Clients attending these services usually have addictions of relatively long-standing and/or high severity (see Deane, Kelly, Crowe, Coulson \& Lyons, 2013 for normative comparisons on ASI). They also have high levels of comorbid mental health disorders (approximately 74\%, Mortlock, Deane \& Crowe, 2011). Almost all have previously attempted less intensive forms of treatment such as outpatient services or self-help groups. The program incorporates group therapy sessions, individual 
case management and attendance at chapel. The group therapy provided during the program covers a wide range of domains including social and communication skills training, components of psycho-education, motivational enhancement, self-esteem work, relapse prevention and anger management.

Participants

Participants were selected from a cohort of clients admitted to the centres for treatment during the period of June 2008 to July 2010 inclusive. The potential participant pool was 1452. Selected participants met two additional criterion: (i) they had provided informed consent for participation in the study and (ii) they had completed the Recovery Assessment Scale as part of the formal intake assessment $(\mathrm{N}=1094)$. This provided a participation rate of $75.34 \%$ at baseline. A total of 374 participants were able to be contacted at 3-month follow-up and completed the follow-up interview and measures. This provided a follow-up rate of 34.18\%. Demographic characteristics of the sample and mean LOS are presented in Table 1.

Insert Table 1 here

\section{Measures}

The Addiction Severity Index $5^{\text {th }}$ ed. (ASI; McLellan et al., 1992). The ASI is a semistructured interview used to determine the severity of an individual's health status in seven domains: Medical Status, Employment/Support Status, Alcohol Use and Drug Use, Legal Status, Family/Social Relationships and Psychiatric Status. A composite score is generated from the items in each domain which determines the overall problem severity in that area. Previous research has shown the ASI had Cronbach’s alphas of .91 (alcohol use) and .71 (drug use) (Bovasso et al., 2001). The current study utilised the alcohol and drug composites only and had Cronbach’s alphas of .89 and .73 respectively. 
The Depression, Anxiety and Stress Scale - 21 (DASS-21; Lovibond \& Lovibond, 1995). The DASS-21 is a 21 item self-report scale that measures a person's affective states of depression, anxiety and stress. Prior research has shown the DASS-21 subscales to have good concurrent validity with other well established depression and anxiety measures (Beck Depression Inventory $r=.79$ ), (Beck Anxiety Inventory, $r=.85$ ), In the present study, the total scale was used and the Cronbach’s alpha was .95.

Recovery Assessment Scale (RAS; Corrigan et al., 2004). The RAS is a 24- item selfreport measure used to derive client perceived assessment of recovery across five factors: personal confidence and hope, willingness to ask for help, goal success and orientation, reliance on others and no domination by symptoms. The RAS has a Cronbach's alpha of .87 (personal confidence and hope), .84 (willingness to ask for help), .82 (goal success and orientation) and .74 (reliance on others and no domination by symptoms) (McNaught et al., 2007). In the current study the Cronbach’s alpha for the full scale was .91.

Mental Health Continuum-Short Form (MHC-SF; Lamers et al., 2011). The MHC-SF is a 14-item self-report scale which measures an individual's emotional, psychological and social well-being. Previous research has shown the MHC-SF to have good internal consistency (>.80) and discriminant validity in adults and adolescents (Lamers et al., 2011; Westerhof and Keyes, 2010). The test-retest reliability of the MHC-SF over three successive 3 month periods averaged .68 and a 9 month test-retest was .65 (Lamers et al., 2011). In the present study the Cronbach’s alpha for the full scale was .94.

The Life Engagement Test (LET; Scheier et al., 2006): The LET is a six-item selfreport questionnaire that measures an individual's purpose in life, defined in terms of the degree to which a person engages in personally valued activities. Previous research has found the LET to have a Cronbach's alpha of 0.80 (Scheier et al., 2006). In the present study the Cronbach’s alpha was .72. 


\section{Procedure}

Upon admission to the program Salvation Army staff perform a routine intake interview using the ASI and questionnaires. The Salvation Army staff (centre managers and clinical employees) were trained in the administration of all outcome measures used in the study by the research team. Those clients providing informed consent completed all measures during this intake session. The Salvation Army staff entered data into the Salvation Army's online Service and Mission Information System (SAMIS) and data was transferred to a deidentified electronic file for analysis by the research team. Three month post-discharge follow-up interviews were conducted at the University of Wollongong via phone, by trained psychology research assistants. Participants were provided with an AUD\$20 gift voucher for follow-up interview completion. The research received ethical review and approval from the University of Wollongong Human Research Ethics Committee.

\section{Analytic Strategy}

The Christensen and Mendoza (1986) formula was used to calculate reliable change indices (RCI). It is proposed that in order for reliable change to occur, a participant's difference in total score from intake to follow up must be equal to or greater than the RCI calculated for each measure. Group membership (reliable deterioration/not improved or reliably improved) was determined using the above method. The cut-off points calculated for reliable change on the outcome measures were as follows: DASS, 7.08, LET, 5.19, MHC-SF, 2.25, RAS, 2.50 .

Following calculations of reliable change indices, binominal logistic regression analyses were conducted using the outcome measures with the dichotomous dependent variables of "reliably improved" and "not improved". Those allocated to the "not improved" category comprised participants who had experienced no change and the small number who had experienced reliable deterioration (range $\mathrm{N}=29$ to 49). The number of matched intake 
and follow-up participant data that were available for logistic regression analyses were:

DASS $N=131$, LET $N=150$, MHC-SF $N=112$, RAS, $N=150$. LOS, gender, age, primary substance of abuse (alcohol vs. other) and ASI alcohol and drug problem severity comprised the independent variables.

\section{Results}

\section{Attrition Analyses}

Independent sample t-tests and chi-square tests of contingencies were performed, comparing between group differences on baseline variables (gender, age, education level, ethnicity, primary substance use, the DASS, RAS, MHC-SF and LET) for those who completed follow up and those who did not. Those who completed follow up were significantly older $(\mathrm{M}=37.40, \mathrm{SD}=10.70, \mathrm{~N}=353)$ than non-completers $(M=35.16, \mathrm{SD}=$ 10.30, $N=938) t(1289)=-3.47, p=.001$. A similar finding was observed for LOS with follow-up completers $(M=112.30, S D=94.75, N=354)$ demonstrating longer stays than their non-completing counterparts $(M=95.80, S D=83.44, N=938), t(1290)=-2.88, p=$ .004. There were no further significant between groups differences,.

Intake to 3-month post-discharge outcomes

Paired sample t-tests revealed significant intake to follow-up improvement across all four measures: DASS intake $(M=57.54, S D=30.58)$ follow-up $(M=33.68, S D=27.20) t(268)=$ 10.78,; LET intake $(M=3.37, S D=.67)$ follow-up $(M=3.53, S D=.75), t(285)=-2.94$; MHC-SF intake $(M=2.40, S D=1.15)$ follow-up $(M=3.09, S D=1.09) t(230)=-7.42$; RAS intake $(M=3.60, S D=.59)$ follow-up $(M=3.84, S D=.63) t(296)=-5.39$, all $p=<.05$. There were variations in the sample sizes for different measures due to some participants not completing all measures. Table 2 provides the proportions of participants who were reliably improved, not improved or reliably deteriorated. 
Relationship between outcome difference scores and LOS

Pearson's correlation coefficients $(r)$ were calculated between LOS and changes on outcome measures. LOS was significantly correlated with baseline to follow-up difference scores on the DASS $(r=-.21)$, LET $(r=.17)$, RAS $(r=.23)$ and MHC-SF $(r=.21$, all $p<$ $.01)$.

\section{Evaluation of differences in LOS between RCI groups}

Independent samples t-tests assessed the differences in LOS between RCI groups and outcome measures. Significant between group differences were observed for all four measures (Table 2). The "reliably improved” group had significantly longer LOS than those in the "not improved” group on all measures. Overall, those designated as reliably improved stayed on average 37.37 days longer than those who made no improvement.

Relationship between RCI groups and primary substance of abuse

Chi-square tests were performed between RCI groups and primary substance of abuse groups “alcohol” vs. “all other substances”. Chi-square was statistically significant for the RAS only, $\chi^{2}(1, N=260)=6.92, p=.009$. Those in the "all other substances" group had a higher proportion of participants classified as reliably improved (41\%) with regard to client perceived recovery compared to the "alcohol” group (25\%).

Predicting reliable improvement

A series of binomial logistic regressions were calculated to predict reliably improved or no improvement group membership on all outcome measures. Predictor (IVs) in all models were: LOS, Primary substance of abuse (Alcohol vs. Other), Age group (17-29, 30-43, 4468 ) and gender. The full model was not significant for the DASS $\chi^{2}(n=131)=11.10, d f=6$, $p=.134$, Nagelkerke $\mathrm{R}^{2}=.11$ or the $\operatorname{LET} \chi^{2}(\mathrm{n}=150)=11.02, \mathrm{df}=6, p=.138$, Nagelkerke $\mathrm{R}^{2}$ $=.11$. 
The full model containing all predictors was statistically significant for well-being (MHC-SF), $\chi^{2}(\mathrm{n}=112)=15.98, \mathrm{df}=6, p=.025$. The strength of the association was calculated as Nagelkerke $\mathrm{R}^{2}=.18$. The model correctly classified $62.8 \%$ of cases $(70.5 \%$ correctly classified as making reliable improvement and 53.8\% correctly classified as making no improvement). LOS was the only significant predictor. The odds of being reliably improved increased 2.24 times with every 90 days spent in treatment. Primary substance of abuse only approached significance, but suggested that those with “alcohol” as their primary problem were almost four times more likely to be reliably improved compared to those with “all other substances” as their primary problem, $\operatorname{Exp}(\beta)=3.78$.

The full model was statistically significant for client perceived recovery (RAS), $\chi^{2}$ $(\mathrm{n}=150)=16.48, \mathrm{df}=6, p=.021$. The strength of the association was calculated as Nagelkerke $\mathrm{R}^{2}=.18$. The model correctly classified $73.3 \%$ of cases $(21.4 \%$ correctly classified as making reliable improvement and 93.5\% correctly classified as making no improvement). LOS was the only significant predictor. Subsequently, the odds of being reliably improved increased 1.57 times with every 90 days spent in treatment.

\section{Discussion}

This study aimed to establish LOS in a residential treatment program for substance abusers as a predictor of reliable change across a range of psychological outcome measures. Reliable change was observed across all four measures from intake to follow-up. The RCI indicated that between $19.9 \%$ and $67.7 \%$ of clients demonstrate reliable change. Regression analyses confirm LOS as predictor of reliable change above other potential predictors on two of the outcome variables examined. It was found that for social, emotional and psychological well-being and client perceived assessment of recovery, each 90 day interval spent in treatment translated to a significant increase in the likelihood of making reliable change. These results extend upon the 'threshold' theory that consumers must spend at least 90 days 
in treatment for positive outcomes to occur (Simpson et al., 1999). Essentially, these findings indicate, that reliable change on wellbeing and recovery is between 1.5 and 2.5 times more likely to occur at the 90 day threshold. However, each 90 day period beyond this point increases the probability of reliable change to a similar increment.

Linear relationships have previously been reported between LOS and statistically significant outcomes (Gossop et al., 1999). However, this study established a linear relationship between LOS and reliable change taking into account measurement error. Higher rates of reliable change do appear related to at least 90 day stays, however, the probability of this increases significantly with longer tenure. A major consideration for treatment providers, is whether longer programs (which increase the likelihood of reliable change) are feasible in the face of evidence to suggest that reliable change is probable (albeit, slightly less so) in shorter time frames.

Membership in the 'alcohol' as primary substance of abuse category approached significance for predicting reliable change in well-being. It is possible, due to social and legal acceptance and a culture which permits heavy episodic drinking, that alcohol is a more insidious drug of misuse (Deane et al., 2012). Given this, an individual may engage in problematic drinking for a considerable period before damaging social relations. Thus, recovery capital components such as community group membership and family support may be more likely to be intact. Consequently, it is possible to speculate the alcohol user is more likely to be socially connected and more likely to endorse statements in the MHC-SF such as "you belonged to a community (like a social group, or your neighbourhood)" than the individual engaging in other less accepted drug use.

As expected, there was a significant difference in LOS between RCI groups, with those categorised as reliably improved demonstrating longer stays than those designated as not improved. Those in the reliably improved groups tended to stay on average 4 months and 
2 weeks compared to just under 3 months for those in the not improved category. This finding suggests the difference in LOS between those who were reliably improved or not improved is just over 1 month. Identification of this critical juncture is an important finding in the context of retaining clients in treatment and treatment planning.

The present study has several limitations. Firstly, the follow-up rate of $34 \%$ is low, as is the completion rate of some of our measures, meaning our results are to be interpreted with some degree of caution. This attrition may pose a threat to the internal and external validity of our findings. A prior analysis comparing those lost to follow-up with those who completed follow-up in the same services as in this study indicated no pre-treatment differences between the groups (Deane, Kelly, Crowe, Lyons \& Cridland, 2014). Our analyses of attrition indicated between groups differences in age and LOS only, with those completing follow-up being on average 2 years older and staying in treatment 16.5 days longer. Thus, some care should be exercised in generalising the results to younger samples or those with shorter treatment durations.

The inclusion of only one service type is also a limitation of this study. Future research efforts may wish to include analyses of clients from different treatment modalities to assess reliable change across varied models of substance abuse interventions. With larger samples there may be the opportunity to provide more detailed drug type typologies that also include multiple forms of poly-drug use. Similarly larger sample sizes will allow further clarification about whether no change groups and deteriorated groups have differential lengths of stay.

Overall, this study suggests that clients who participated in residential substance abuse treatment experienced reliable change on all four measures of psychological well-being and recovery. Only minimal differences were noted with regards to outcome based on primary drug problem. The LOS difference between those who were reliably improved vs. 
not improved was just over 1 month, indicating an important juncture for service providers in relation to treatment retention. Furthermore, LOS emerged as an independent predictor of reliable change on two of the four measures included in this study. This highlights the utility of LOS in predicting client outcomes with regard to psychological recovery. 


\section{References}

Bovasso, G. B., Alterman, A. I., Cacciola, J. S., \& Cook, T. G. (2001). Predictive validity of the Addiction Severity Index's composite scores in the assessment of 2-year outcomes in a methadone maintenance population. Psychology of Addictive Behaviors, 15, 171-176.

Christensen, L., \& Mendoza, J. L. (1986). A method for assessing change in a single subject: An alteration of the RC index. Behaviour Therapy, 17(3), 305 - 308.

Choi, S., Adams, S. M., Morse, S. A., \& MacMaster, S. (2015). Gender differences in treatment retention among individuals with co-occurring substance abuse and mental health disorders. Substance Use \& Misuse, 50(5), 653 - 663.

Condelli, W. S., \& Hubbard, R. L. (1994). Relationship between time spent in treatment and client outcomes from therapeutic communities. Journal of Substance Abuse Treatment, 11(1), 25 33.

Corrigan, P. W., Salzer, M., Ralph, R. O., Sangster Y., \& Keck, L. (2004). Examining the factor structure of the Recovery Assessment Scale. Schizophrenia Bulletin, 30(4), 1035 - 1041.

Darke, S., Campbell, G., \& Popple, G. (2012). Retention, early dropout and treatment completion among therapeutic community admissions. Drug and Alcohol Review, 31, 64 - 71.

Deane, F. P., Kelly, P. J., Crowe, T. P., Coulson, J., \& Lyons, G. (2013). Clinical and Reliable Change in an Australian Residential Substance Use Program Using the Addiction Severity Index. Journal of Addictive Diseases, 32, 194 - 205.

Deane, F. P., Kelly, P. J., Crowe, T. P., Lyons, G. C. B., \& Cridland, L. E., (2014). Feasibility of telephone follow-up interviews for monitoring treatment outcomes of Australian residential drug and alcohol treatment programs. Substance Abuse, 35, 21 - 29.

Deane, F. D., Wootton, D. J., Hsu, C., \& Kelly, P.J. (2012). Predicting dropout in the first 3 months of 12-step residential drug and alcohol treatment in an Australian sample. Journal of Studies on Alcohol and Drugs, 73, $216-225$. 
del Vecchio, P. (2012, March 23). SAMAHA’s working definition of recovery updated.

[Web blog post]. Retrieved from http://blog.samhsa.gov/2012/03/23/defintion-of-recoveryupdated/

Eisen, S. V., Ranganathan, G., Seal, P., \& Spiro, A. (2007). Measuring clinically meaningful change following mental health treatment. The Journal of Behavioural Health Services and Research, 34(3), 272 - 289.

Ernst and Young (1996). Review of long-term residential treatment for people with alcohol and other drug use problems. Commonwealth Department of Human Services and Health: Canberra.

Gonda, T., Deane, F. P., \& Murugesan, G. (2012). Predicting clinically significant change in an inpatient program for people with severe mental illness. The Australian and New Zealand Journal of Psychiatry, 46(7), 651 - 658.

Gossop, M. (1995). The treatment mapping survey: A descriptive study of drug and alcohol treatment responses in 23 countries. Drug and Alcohol Dependence, 39, 7 - 14.

Gossop, M., Marsden, J., Stewart, D., \& Rolfe, A. (1999). Treatment retention and 1 year outcomes for residential programmes in England. Drug and Alcohol Dependence, 57, 89 - 98.

Green, C. A., Polen, M. R., Lynch, F. L., Dickinson, D. M., \& Bennett, M. D. (2004). Gender differences in outcomes in an HMO-based substance abuse treatment program. Journal of Addictive Diseases, 23, 47 - 70.

Greenfield, L., Burgdorf, K., Chen, X., Porowski, A., Roberts, T., \& Herrell, J. (2004). Effectiveness of long-term residential substance abuse for women: Findings from three national studies. American Journal of Drug and Alcohol Abuse, 30, 537 - 550.

Hambley, J., \& Arbour, S. (2010). Comparing outcomes for alcohol and drug abuse clients: A 6month follow-up of clients who completed a residential treatment program. Journal of Substance Use, 15(3), $184-200$. 
Lai, H. M., Cleary, M., Sitharthan, T., \& Hunt, G. E. (2015). Prevalence of comorbid substance use, anxiety and mood disorders in epidemiological surveys, 1990-2014: A systematic review and meta-analysis. Drug and Alcohol Dependence, 154, 1 - 13.

Lamers, S. M. A., Westerhof, G. J., Bohlmeijer, E. T., ten Klooster, P. M., \& Keyes, C. L. M. (2011). Evaluating the psychometric properties of the Mental Health Continuum- Short Form (MHC-SF). Journal of Clinical Psychology, 67(1), 99 - 110.

Lovibond, P. F., \& Lovibond, S. H. (1995). The structure of negative emotional states: Comparison of the Depression Anxiety Stress Scales (DASS) with the Beck Depression and Anxiety Inventories. Behaviour Research and Therapy, 33, 335 - 343.

McLellan, T., Kushner, H., Metzger, D., Peters, R., Smith, I., Grissom, G. ... Argeriou, M. (1992). The fifth edition of the Addiction Severity Index. Journal of Substance Abuse Treatment, 9(3), $199-213$.

McNaught, M., Caputi, P., Oades, L, G., \& Deane, F. P. (2007). Testing the validity of the Recovery Assessment Scale using an Australian sample. The Australian and New Zealand Journal of Psychiatry, 41, 450 - 457.

Mertens, J. R., \& Weisner, C. M. (2000). Predictors of substance abuse treatment retention among men and women in an HMO. Alcoholism: Clinical and Experimental Research, 24(10), 1525 $-1533$.

Miller, N. S., Millman, R. B., \& Keskinen, S. (1990). Outcome at six and twelve months post inpatient treatment for cocaine and alcohol dependence. Advances in Alcohol Substance Abuse, 9(3-4), $101-120$.

Mortlock, K., Deane, F. P., \& Crowe, T. P. (2011). Screening for mental disorder comorbidity in Australian alcohol and other drug residential treatment Settings. Journal of Substance Abuse Treatment, 40, $397-404$. 
Mulder, R. T., Frampton, C. M. A., Peka, H., Hampton, G., \& Marsters, T. (2009). Predictors of 3month retention in a drug treatment therapeutic community. Drug and Alcohol Review, 28, $366-371$.

Newnham, E. A., Harwood K. E., \& Page, A. C. (2007). Evaluating the clinical significance of responses by psychiatric inpatients to the mental health subscales of the SF-36. Journal of Affective Disorders, 98(1-2), 91 - 97.

Ryan, R. M., Plant, R. W., \& O'Malley, S. (1995). Initial motivations for alcohol treatment: Relations with patient characteristics, treatment involvement, and dropout. Addictive Behaviors, 20(3), 279 - 297.

Satre, D. D., Mertens, J. R., Arean, P. A., \& Weisner, C. (2004). Five-year alcohol and drug treatment outcomes of older adults versus middle-aged and younger adults in a managed care program. Addiction, 99(10), 1286 - 1297.

Scheier, M. F., Wrosch, C., Baum, A., Cohen, S., Martire, L. M., Matthews, K. A. ... Zdaniuk, B. (2006). The life engagement test: Assessing purpose in life. Journal of Behavioral Medicine, 29, $291-298$.

Simpson, D. D., Joe, G. W., \& Brown, B. S. (1997). Treatment retention and follow-up outcomes in the drug abuse treatment outcome study (DATOS). Psychology of Addictive Behaviours, 11(4), $294-307$.

Simpson, D. D., Joe, G. W., Fletcher, B. W., Hubbard, R. L., \& Anglin, D. M. (1999). A national evaluation of treatment outcomes for cocaine dependence. Archives of General Psychiatry, 36, $507-515$

Teeson, M., Marel, C., Darke, S., Ross, J., Slade, T., Burns, L... Mills, K. L. (2015). Long- term mortality, remission, criminality and psychiatric comorbidity of heroin dependence: 11-year findings from the Australian Treatment Outcome Study. Addiction, 110(6), 986 - 983. 
Teeson, M., Mills, K., Ross, J., Darke, S., Williamson, A., \& Havard, A. (2007). The impact of treatment on 3 years’ outcome for heroin dependence: Findings from the Australian Treatment Outcome Study (ATOS). Addiction, 103(1), 80 - 88.

Weisner, C., Delucchi, K., Matzger, H., \& Schmidt, L. (2003). The role of community services and informal support on five-year drinking trajectories of alcohol dependent and problem drinkers. Journal of Studies on Alcohol, 64, 862 - 873.

Westerhof, G. J., \& Keyes, C. L. M. (2010). Mental illness and mental health: The two continua model across the lifespan. Journal of Adult Development, 17, 110 - 119.

Zhang, Z., Friedmann, P. D., \& Gerstein, D. R. (2002). Does retention matter? Treatment duration and improvement in drug use. Addiction, 98, $673-684$. 
Table 1. Demographic characteristics and length of stay for the current sample

\begin{tabular}{|c|c|c|c|c|c|c|c|c|}
\hline & \multirow[t]{2}{*}{ All } & \multirow[t]{2}{*}{ Male } & \multirow[t]{2}{*}{ Female } & \multirow[t]{2}{*}{ Alcohol } & \multirow{2}{*}{$\begin{array}{l}\text { All Other } \\
\text { Substances }\end{array}$} & \multirow[t]{2}{*}{ Age 17-29 } & \multirow[t]{2}{*}{ Age 30-43 } & \multirow[t]{2}{*}{ Age 44-68 } \\
\hline & & & & & & & & \\
\hline & $N=374$ & $N=305$ & $N=69$ & $N=172$ & $N=95$ & $\mathrm{~N}=100$ & $\mathrm{~N}=160$ & $\mathrm{~N}=96$ \\
\hline \multicolumn{9}{|l|}{ Gender } \\
\hline \% Male & $81.7 \%$ & - & - & $80.8 \%$ & $82.1 \%$ & $80.0 \%$ & $82.5 \%$ & $82.3 \%$ \\
\hline Anglo & $88.5 \%$ & $89.5 \%$ & $85.1 \%$ & $91.8 \%$ & $86.2 \%$ & $92.0 \%$ & $88.2 \%$ & $84.4 \%$ \\
\hline Other & $11.5 \%$ & $10.5 \%$ & $14.9 \%$ & $8.2 \%$ & $13.8 \%$ & $8.0 \%$ & $11.8 \%$ & $15.6 \%$ \\
\hline \multicolumn{9}{|l|}{ Education } \\
\hline Primary & $1.1 \%$ & $1.0 \%$ & $1.5 \%$ & - & $2.1 \%$ & $1.0 \%$ & $0.7 \%$ & $1.0 \%$ \\
\hline \multicolumn{9}{|l|}{ Substance } \\
\hline Alcohol & $64.4 \%$ & $64.1 \%$ & $66.7 \%$ & - & - & $33.8 \%$ & $69.4 \%$ & $89.6 \%$ \\
\hline All other & $35.6 \%$ & $35.9 \%$ & $33.3 \%$ & - & - & $66.2 \%$ & $30.6 \%$ & $10.4 \%$ \\
\hline LOS M(SD) & 109.82(93.81) & 113.08(95.53) & $97.00(85.82)$ & $117.48(96.46)$ & 107.86(90.98) & 102.63(95.19) & 106.64(88.11) & 116.22(99.03) \\
\hline
\end{tabular}


Table 2. Proportion of participants who experienced reliable change and differences in length of stay (days) between groups

\begin{tabular}{|c|c|c|c|c|c|c|c|}
\hline \multirow[b]{2}{*}{ Measure } & \multicolumn{3}{|c|}{ Total Sample } & \multicolumn{2}{|c|}{ Length of Stay (days) } & \multirow[b]{2}{*}{$\mathrm{t}$} & \multirow[b]{2}{*}{$\begin{array}{l}\text { Mean difference in } \\
\text { LOS for RI } \\
(\mathrm{M}=37.37)\end{array}$} \\
\hline & $\begin{array}{l}\text { \% Reliably } \\
\text { Improved } \\
\text { (n) }\end{array}$ & $\begin{array}{l}\text { \% Not } \\
\text { Improved } \\
\text { (n) }\end{array}$ & $\begin{array}{l}\text { \% Reliably } \\
\text { Deteriorated } \\
\text { (n) }\end{array}$ & $\begin{array}{l}\text { Reliably } \\
\text { Improved } \\
\mathrm{M}(\mathrm{SD})\end{array}$ & $\begin{array}{l}\text { Not Improved/ } \\
\text { Deteriorated } \\
\mathrm{M}(\mathrm{SD})\end{array}$ & & \\
\hline DASS & $67.7(182)$ & $14.1(38)$ & $18.2(49)$ & 129.52(96.99) & $90.10(77.83)$ & $-3.30 *$ & 39.42 \\
\hline LET & $19.9(57)$ & $63.6(182)$ & $16.4(47)$ & 150.37(101.27) & 105.39(88.43) & $-3.39 *$ & 44.98 \\
\hline MHC-SF & $42.4(98)$ & $45.0(104)$ & $12.6(29)$ & 102.63(79.29) & 73.62 (59.96) & $-3.16 *$ & 29.01 \\
\hline RAS & $31.9(94)$ & $53.6(158)$ & $14.6(43)$ & 125.60(98.56) & $89.53(74.21)$ & $-3.15 *$ & 36.07 \\
\hline
\end{tabular}

Note. DASS, Depression Anxiety and Stress Scale. LET, Life Engagement Test. MHC-SF, Mental Health Continuum- Short Form. RAS,

Recovery Assessment Scale. ${ }^{*} p<.05$ for comparisons between groups on LOS 